\title{
Unique Secreted in Xylem Genes in Banana-Infecting Endophytic Fusarium Oxysporum ${ }^{+}$
}

\author{
Rebecca Lyons *, Elizabeth Czislowski, Isabel Zeil-Rolfe, Shubhdeep Kaur, Zhendong Liu, \\ Andy Chen and Elizabeth Aitken \\ School of Agriculture and Food Sciences, The University of Queensland, Brisbane 4072, Australia; \\ Elizabeth.Czislowski@daf.qld.gov.au (E.C.); isabel.zeilrolfe@uq.net.au (I.Z.-R.); \\ shubhdeep.kaur@uq.net.au (S.K.); hendong.liu@uq.net.au (Z.L.); a.chen2@uq.edu.au (A.C.); \\ e.aitken@uq.edu.au (E.A.) \\ * Correspondence: r.lyons@uq.edu.au \\ + Presented at the third International Tropical Agriculture Conference (TROPAG 2019), Brisbane, Australia, \\ 11-13 November 2019.
}

Published: 7 April 2020

\begin{abstract}
Members of the Fusarium oxysporum species complex include pathogenic and nonpathogenic isolates and infect a broad range of plant species. F. oxysporum f. sp. cubense (Foc) causes the destructive Fusarium wilt of banana, and the recently emerged Foc tropical race 4 strain threatens the global banana industry. Secreted in xylem (SIX) genes encode for F. oxysporum effector proteins that are associated with virulence in pathogenic F. oxysporum, however they have rarely been reported from non-pathogenic $F$. oxysporum isolates. Our recent survey of asymptomatic banana plants grown in Foc-infested fields in Queensland and northern NSW revealed that diverse Fusarium spp, including F. oxysporum, reside in the plant roots and pseudostem without causing obvious damage to the plant. Intriguingly, we amplified SIX genes from several of the putative endophytic F. oxysporum isolates identified in the survey and found that they differ in their profile to known Foc SIX genes. To study the role of the endophytic F. oxysporum isolates in planta and the biological function of their SIX genes in more detail, we will re-inoculate cultivated and wild diploid banana lines with the endophytic F. oxysporum strains under glasshouse conditions to assess if they are non-pathogenic on banana. Secondly, we will determine whether the endophytic $F$. oxysporum SIX genes are expressed in planta and/or in vitro and look at the transcriptome changes occurring in the host following infection. Finally, endophytic F. oxysporum strains transformed with GFP will be used to investigate the extent of fungal colonisation in the plant.
\end{abstract}

Keywords: plant immunity; endophytes; fungal disease; banana; Fusarium oxysporum

Author Contributions: R.L., E.C., A.C. and E.A. conceived and planned the experiments; R.L., E.C., I.Z.-R., S.K. and Z.L. performed the experiments, E.A. supervised the project. All authors have read and agreed to the published version of the manuscript.

Funding: Horticulture Innovation Australia BA16005.

Conflicts of Interest: The authors declare no conflict of interest.

(C) 2020 by the authors. Licensee MDPI, Basel, Switzerland. This article is an open access article distributed under the terms and conditions of the Creative Commons Attribution (CC BY) license (http://creativecommons.org/licenses/by/4.0/). 